\title{
An Exploration Strategy for Europa
}

Corresponding Author: Cynthia B. Phillips, Jet Propulsion Laboratory, California Institute of Technology; cynthia.b.phillips@jpl.nasa.gov

Co-Authors: Kevin P. Hand ${ }^{1}$, Jennifer Scully ${ }^{1}$, Jo Pitesky ${ }^{1}$, Kate Craft ${ }^{2}$, Marissa E. Cameron ${ }^{1}$, Tom Nordheim ${ }^{1}$, Amy E. Hofmann ${ }^{1}$, Grace Tan-Wang ${ }^{1}$, Jason D. Hofgartner ${ }^{1}$, Morgan Cable ${ }^{1}$

Co-signers: Charity Phillips-Lander ${ }^{3}, \mathrm{Jim}_{\text {Burch }}^{3}$, Ross Beyer, ${ }^{4,33}$, Everett Shock ${ }^{5}$, Insoo Jun ${ }^{1}$, Corey Cochrane ${ }^{1}$, Alfred McEwen ${ }^{6}$, Serina Diniega ${ }^{1}$, Hamish Hay $^{1}$, Abigail Rymer ${ }^{2}$, Sierra Ferguson $^{5}$, Angela G. Marusiak ${ }^{1}$, Ashley G. Davies ${ }^{1}$, Jamie Molaro, Erin Leonard ${ }^{1}$, Emily Costello $^{8}$, Edgard Rivera-Valentin ${ }^{9}$, Peter Willis ${ }^{1}$, Sona Hosseini ${ }^{1}$, Samuel Howell ${ }^{1}$, Emily Hawkins $^{10}$, Leonardo Regoli ${ }^{2}$, Maheenuz Zaman ${ }^{11}$, Paul Schenk ${ }^{9}$, Sarah Waller ${ }^{1}$, Akos Kereszturi ${ }^{2}$, Tuan $\mathrm{Vu}^{1}$, Jessica Noviello ${ }^{5}$, Tracy Becker ${ }^{3}$, Mathieu Choukroun ${ }^{1}$, Mohit Melwani Daswani $^{1}$, Richard Mathies ${ }^{13}$, Alex Patthoff ${ }^{7}$, Catherine Walker ${ }^{14}$, Michael Malaska ${ }^{1}$, Marshall Styczinski ${ }^{15}$, Kirsten Sims ${ }^{16}$, Alicia Rutledge ${ }^{17}$, Britney Schmidt ${ }^{18}$, Moses Milazzo ${ }^{19}$, Csilla Orgel $^{20}$, Lynnae C. Quick ${ }^{21}$, Michael C. Nolan ${ }^{6}$, Christopher Bennett ${ }^{22}$, Laura Fackrell ${ }^{23}$, Krista Soderlund ${ }^{24}$, Ali M. Bramson ${ }^{25}$, Yasmina M. Martos ${ }^{21}$, Michael Poston ${ }^{3}$, Alyssa C. Mills ${ }^{26}$, Robert T. Pappalardo ${ }^{1}$, Edith C. Fayolle ${ }^{1}$, Chimira Andres ${ }^{27}$, Geoffrey Collins ${ }^{28}$, Jodi Berdis ${ }^{29}$, Ashley Schoenfeld $^{30}$, Alice Lucchetti ${ }^{31}$, Maurizio Pajola ${ }^{31}$, Jennifer Hanley ${ }^{32}$, Lauren Schurmeier ${ }^{8}$, Heather Smith $^{33}$, Marzia Parisi ${ }^{1}$, Franck Marchis ${ }^{4}$, Ryan Galinkin ${ }^{22}$, Alexis Bouquet ${ }^{34}$, Divya Persaud $^{35}$, Scott Perl ${ }^{1}$, Shawn Brooks ${ }^{1}$, Michael Bramble ${ }^{1}$

1 Jet Propulsion Laboratory, California Institute of Technology; 2 Johns Hopkins Applied Physics Laboratory; 3 SWRI; 4 SETI Institute; 5 Arizona State U.; 6 U. of Arizona; 7 PSI; 8 U. of Hawaii; 9 LPI; 10 Loyola Marymount U.; 11 Lamont-Doherty Earth Observatory, Columbia; 12 Research Centre for Astronomy \& Earth Sciences; 13 UC Berkeley; 14 WHOI; 15 U. Washington; 16 Howard U.; 17 Northern Arizona U.; 18 Georgia Institute of Tech.; 19 Other Orb LLC; 20 ESA-ESTEC; 21 NASA GSFC; 22 U. of Central Florida; 23 U. of Georgia; 24 U. Texas, Austin; 25 Purdue; 26 U. Alabama; 27 U. Western Ontario; 28 Wheaton Coll.; 29 NMSU; 30 UCLA; 31 INAF-OAPD Astro. Obs. Padova; 32 Lowell Obs.; 33 NASA Ames; 34 Aix Marseille U.; 35 University College London

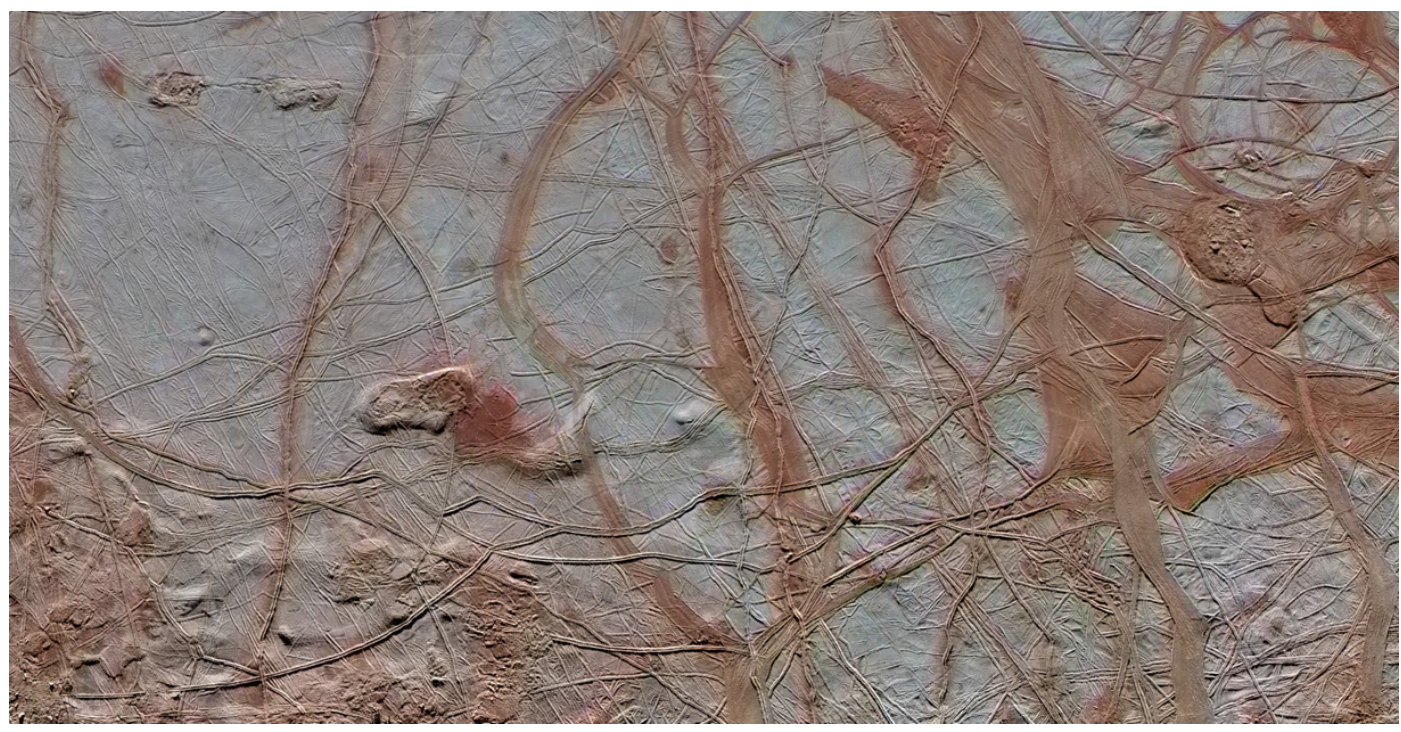

This research was carried out at the Jet Propulsion Laboratory, California Institute of Technology, under a contract with the NASA Aeronautics and Space Administration (80NM0018D0004).

(C) 2020. California Institute of Technology. Government sponsorship acknowledged. 


\section{Executive Summary}

The existence of liquid water in the outer solar system was once thought a remote possibility. However, data from the Galileo spacecraft-geological, gravitational, and magnetic field observations - revealed the likely existence of a global subsurface ocean below Europa's icy surface, with a water volume nearly three times that of Earth's oceans. It is now known that oceans may exist within many large icy moons and potentially even within icy dwarf planets. Understanding and studying these Ocean Worlds is a priority for planetary science and astrobiology.

The exploration of Europa will greatly advance our understanding of Ocean Worlds as possible abodes for life. Europa's relatively thin ice shell $(<30 \mathrm{~km})$, potentially active material exchanges at the surface-ocean and ocean-silicate mantle boundaries, and chemical resources created by surface irradiation within Jupiter's intense radiation environment elevate its priority for astrobiological exploration, and make it a prime example of a potentially habitable Ocean World.

Scientifically, Europa is arguably the premier world in our solar system from which we can: 1) Search for life beyond Earth, including extant life; 2) Understand long-lived global oceans as a planetary process; 3) Understand tidal dissipation in silicate mantles and icy lithospheres; 4) Understand a wide diversity of geological and geophysical processes in globally young ice shells; and 5) Investigate the endogenous chemistry of a subsurface ocean, and the exogenous chemistry resulting from plasma and magnetospheric interactions.

To fully explore Europa and systematically address these investigations, we recommend an integrated, multi-mission program of future Europa exploration. We call for continued support for the Europa Clipper mission, as well as support for follow-on missions including a lander, followed by a probe to explore within the ice shell, and ultimately a probe to directly explore the ocean.

A lander capable of searching for signs of life on the surface of Europa, and providing ground truth for remote sensing and geophysical observations, should be a top flagship priority for the 2023-2032 Decadal Survey.

"Search for life elsewhere" is one of three primary components of the NASA Science Mission Directorate (SMD) mission (NASA, 2020). The importance of the exploration of Europa in this context-as an Ocean World compelling to geology, geophysics, and astrobiology-has been recognized for over 25 years by NASA, NRC committees, and decadal surveys (e.g. COMPLEX 1999, NRC 2003, SSB 2011). The 2003 Decadal Survey, which coincided with the end of the Galileo mission, recommended a Europa Orbiter along with a 'Pathfinder'-type lander and an Astrobiology lander (NRC, 2003).

The most recent Decadal Survey (Space Studies Board, 2011) suggested starting with a remote-sensing mission (hence Europa Clipper), but noted "Ultimately, however, a lander will probably be required to fully characterize organics on the surface of Europa." (Space Studies Board 2011, page 239). We concur with this statement and 
emphasize the ability of a landed mission to answer major questions pertaining to physics, geology, chemistry, and biology, as described in Section 3 below.

The science of Europa is mature, and as Europa Clipper reaches Jupiter we should be prepared to launch a lander to advance the next stage of exploration of this highly compelling target for planetary science and astrobiology.

\section{Previous exploration and state of knowledge}

Ground-based observations showed that Europa's high-albedo surface had the infrared spectrum of water ice (e.g. Kuiper 1957), long before humankind's first close encounter with Europa in 1979. Voyager 2 images revealed a bright surface criss-crossed with long linear features, little topography, and few impact craters, implying a young surface age of $\sim 50$ Ma (Pappalardo et al. 1999; Bierhaus et al., 2009). More detail emerged from images taken from 1996-2002 by the Galileo spacecraft (Figure 1), which showed that the dark "mottled" terrains in Voyager images include regions (now called "chaos" terrain) where the surface ice had been disrupted, broken, and frozen into new positions. These geological observations, along with spectroscopy, gravity measurements, magnetic field measurements, and geological models, strongly suggest that an ocean of liquid water lies beneath Europa's icy surface (Pappalardo et al. 1999).

Figure 1: Most geological models for forming surface features such as (clockwise from top middle) cycloidal ridges, bands, chaos blocks, and double ridges depend on a subsurface liquid water layer. Few impact craters are visible on Europa's surface (left and center), suggesting recent and potentially ongoing resurfacing. (Images from Galileo SSI)

While the surface of Europa is

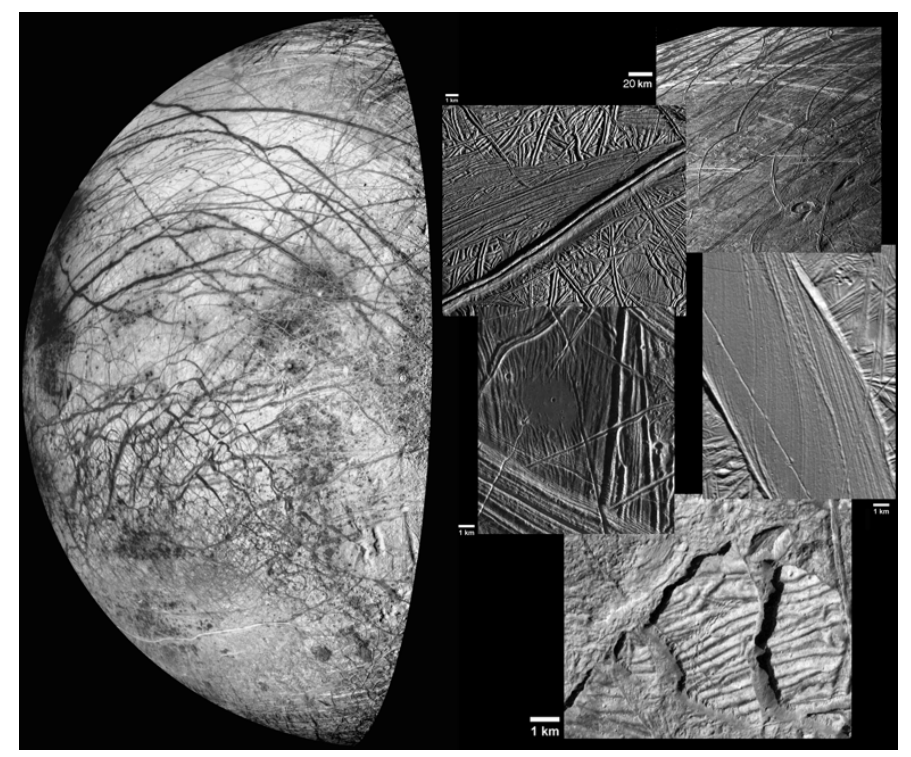
dominated by water ice, the interpretations of "non-ice" features seen spectroscopically by Galileo include the presence of hydrated salts (McCord et al. 2001) or sulfuric acid compounds (Carlson et al. 2005). Gravity measurements also revealed an outer layer roughly $100 \mathrm{~km}$ thick with the density of water or ice (Anderson et al. 1998), but could not distinguish between liquid water and solid ice. Current models usually assume an ice shell thickness between 10-25 km overlaying the liquid water ocean. A thin ice crust could allow transport of radiation-produced oxidants, a possible source of energy for life, from Europa's surface into its ocean, and might also allow transport of ocean materials to the surface. This surface-subsurface exchange would make direct exploration of the ocean easier, and might increase the chances of finding biosignatures on the surface (Chyba and Phillips 2007).

Galileo's observations left behind many new questions about Europa. How thick is the putative ice shell? Is Europa currently geologically active? How does the ocean interact with the ice shell and the underlying rocky layer? What are the compositions of 
Europa's surface and ocean? Are there organic or inorganic biosignatures present on-or near-the surface? Discoveries by the Cassini mission to the Saturn system and groundbased astronomical observations of Europa also led to new questions: Does Europa have plumes emitting subsurface ocean water into space, like Saturn's moon Enceladus? If so, were those plumes active during the Galileo mission, and are they active today? Answers to these questions can only be found by new missions to Europa and the Jupiter system.

\section{Major Unanswered Questions}

Exploring Europa will help us understand the emergence of ocean worlds and habitable worlds in a solar system, whether planets in their own right or planetary satellites. "Searching for life elsewhere" is one of the Core Contexts in the 2018 NASA Strategic Plan (NASA, 2018). NASA's strategic objectives recognize that answering "'Are we alone?' is a central research question that involves biological research and research in the habitability of locations in the solar system such as Mars, the moons of outer planets, or thousands of potentially habitable worlds around other stars. This research supports a fundamental science topic at the interface of physics, chemistry, and biology." The approach we take will stretch beyond a planetary science focus to include biologists, oceanographers, and more (Hand et al., 2020a).

We propose three key unifying themes for studying Europa; our proposed Europa Exploration Strategy comprehensively addresses these themes.

I: Interiors and Origins: Europa and its ocean have likely been in existence for much of the history of the solar system. Radiogenic decay coupled with tidal dissipation (modulated by the beautiful Laplace resonance) have served to sustain this ocean on a timescale commensurate with the age of our own ocean here on Earth (Schubert et al., 2009; Moore and Hussmann, 2009). To this end, we can begin to compare oceans and address questions such as: How did Europa and its ocean form, and how does the physical and chemical oceanography affect its past and present state (e.g., ocean thickness, ocean currents, cycling with the ice shell and seafloor)?

II: Geology and Geophysics: How does Europa evolve, both internally and on its surface? Europa offers a planetary laboratory for investigating and understanding tidal dissipation in silicate mantles and icy lithospheres. The Jovian system is old and the tidal evolution is well-characterized. The energy from tides - dissipated in either the mantle or ice shell - likely drives the diversity of geological and geophysical processes observed globally on Europa. Tectonism, subsumption, convection, and conduction, are just a few of the widescale processes for which Europa offers a second type-example, helping to inform not just how Europa works, but also how these processes work on Earth (Collins et al., 2009; Kattenhorn \& Prockter, 2014). Lastly, as a relatively large and dense Ocean World, Europa retains much of the material that might otherwise be lost to space via plumes or cryovolcanism (Walker et al., 2020). Europa also captures sulfur and other ions from Io, Jupiter, and the solar wind. To this end, Europa's globally young ice shell enables the investigation of the endogenous chemistry of a subsurface ocean, and the exogenous chemistry resulting from plasma and magnetospheric interactions.

III: Biology and Biochemistry: Perhaps the grandest question one can ask about Europa is: Does Europa harbor life? If yes, what regulates the habitability of Europa? If not, but a habitable ocean exists, what are the limits on the origin of life itself? The exploration of Europa's surface and subsurface ocean enables a search for 
biosignatures and possibly even the detection of extant life. The three keystones for life appear to be present on and within Europa (Hand et al., 2009): 1) liquid water is found in the ocean; 2) building blocks such as carbon (in the form of $\mathrm{CO}_{2}$ ), sulfur, and other biologically necessary elements are found on the surface, and likely exist in the interior (Hand et al., 2007; Hansen and McCord, 2008; Carlson et al., 2009); and 3) the energy needed to power life is potentially sustained through a dynamic, reductant-rich waterrock interaction on the seafloor (Vance et al., 2016) that could couple with radiolytically produced oxidants created on the surface, and cycled into the ocean below (Gaidos et al., 1999; Chyba and Phillips, 2001; Hand et al., 2007). Lastly, if evidence of life is found on or within Europa, we can then examine its biochemistry and truly understand a form of life very likely independent of all life on Earth. Does it utilize DNA, RNA, and proteins, or does it function on a much different biochemistry?

\section{Understanding Europa via a Europa Exploration Strategy}

Past missions showed us the uniqueness of Europa and its relevance in the search for life in the Solar System. Now, Europa is ripe for a dedicated exploration strategy via a series of integrated missions, each building on the discoveries of its predecessor (as seen in NASA's very successful Mars exploration strategy).

Europa exploration will also help open another window for studying other Ocean Worlds. The mission types and development sequence, mapped out here for Europa, will be relevant for later exploration of Enceladus and other outer solar system worlds. The timescales for missions to the outer Solar System are longer than in the inner Solar System, and the costs are higher. Therefore, we suggest that scientifically, it makes sense to think of a Europa exploration campaign that is similar to the current coordinated suite of Mars missions, but in this case enabling the exploration of all Ocean Worlds.

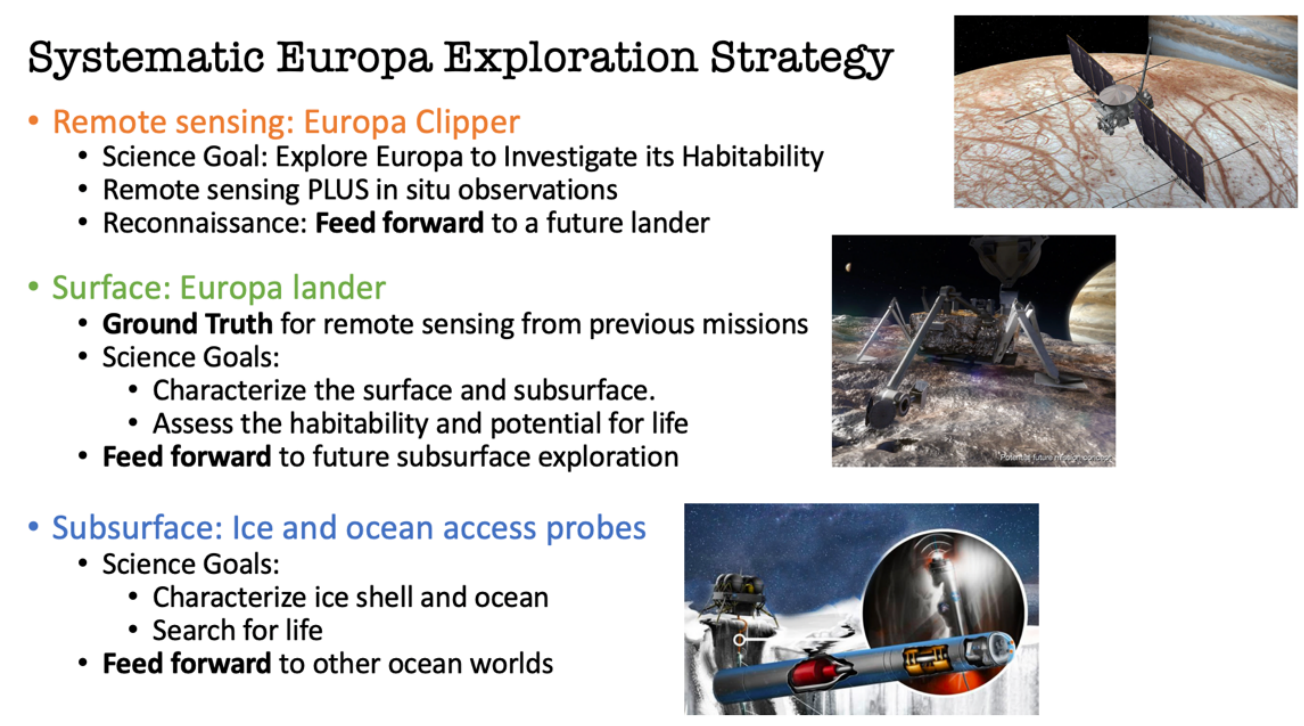

\subsection{Step 1: Remote Sensing (Europa Clipper and JUICE)}

Any Europa exploration strategy begins with a dedicated orbiter or multiple flyby mission, to perform global reconnaissance and start to answer many of the questions listed in Section 3. Galileo imaged only about $10 \%$ of Europa's surface at a resolution of $200 \mathrm{~m} /$ pixel or higher, with very limited coverage taken at resolutions as high as 6-10 
$\mathrm{m} /$ pixel (Greeley et al. 2000). With a planned launch date in the mid 2020's, the NASA Europa Clipper mission will investigate Europa's habitability by considering three key science objectives: studying Europa's ice shell and ocean, its composition, and its geology. Europa Clipper will orbit Jupiter, making about 50 close flybys of Europa. Its scientific payload includes remote sensing instruments, as well as a sounding radar and gravity science investigations and the capability for in situ analysis of material ejected off the surface into space (Pappalardo et al., 2020).

The Europa Clipper mission will enable landing site selection for a lander. Global imaging and geological mapping at mid-to-high resolution will allow selection of candidate landing sites that are geologically young, located in interesting terrain units, and smooth and free of landing hazards. Spectroscopic data taken from orbit will help select candidate landing sites located in areas where non-ice components are present and could be sampled directly and studied by a lander. Europa Clipper will also search for regions of recent or current geologic activity, hotspots, or plumes, all of which could be relevant locations for future landed exploration.

The European Space Agency's JUICE (JUpiter ICy moons Explorer) mission is also headed for launch in the mid-2020's. JUICE will perform a few close flybys of Europa and Callisto before entering orbit around Ganymede, Jupiter's largest moon and the main scientific target of the mission. JUICE will carry a payload similar to Europa Clipper's, with the addition of a laser altimeter to map the topography of Ganymede.

\subsection{Step 2: Surface Lander}

Answering the rest of the questions identified in the three themes above requires measurements that can only be made from — or beneath — the surface. For example, while orbital data could survey the astrobiological potential of Europa for organic and inorganic biosignatures, only a lander could carry out definitive, direct detection of life or its biosignatures, via multiple robust and complementary measurements (Europa Lander SDT Report, 2016; Hand et al., 2020a, b). A lander could also identify the in situ composition of the surface and its physical state, including the location and physical state of various constituents; determine how physical properties vary with depth; and assess the surface grain size, porosity, and texture. Precise geophysical data that tell us more about Europa's interior and structure - such as seismic measurements - must be gathered from the surface. Other measurements, such as direct sampling of liquid water, would require a subsurface probe. Clearly, fully exploring Europa as an Ocean World calls for a suite of future missions including landers and probes for direct ocean exploration.

The next step in Europa exploration following Europa Clipper should be a landed mission, such as the Europa Lander Mission Concept (Hand et al., 2020b; Europa Lander Mission Concept Resources). The top-level science goals of the Europa Lander are to search for biosignatures, study the habitability of Europa using in situ techniques, and determine the geological and geophysical context of the surface and subsurface.

The Europa Lander mission concept utilizes many technologies developed for Mars landings and in situ surface science. Once on the surface, the sampling system would collect at least 3 samples from a minimum of $10 \mathrm{~cm}$ depth below Europa's surface. Samples could then be analyzed by a variety of instruments located inside a radiationshielded vault. The model science payload also includes a camera, and a seismic package 
for subsurface characterization. In addition to the exciting possibility of directly studying potential biosignatures, a Europa Lander would also provide in situ verification of the remote sensing data from Europa Clipper and other missions (as well as telescopic observations), establishing fundamental ground truth for icy worlds.

\subsection{Step 3: Subsurface Exploration}

On Mars, our exploration paradigm has been flyby $\rightarrow$ orbit $\rightarrow$ land $\rightarrow$ rove, but on an Ocean World like Europa, the most interesting direction of mobility may be vertical rather than horizontal. An initial subsurface probe could target water inclusions located completely within the ice crust, if found by Europa Clipper's ice penetrating radar or by a landed mission. The grand goal of subsurface exploration is placing a mobile cryobot into Europa's ocean via an ocean access probe that drills or melts through the entire surface ice layer (e.g. Oleson et al., 2019, Balint et al., 2020). Such a mission would allow direct access to the ocean itself, and would provide the best way to directly observe potentially habitable, or inhabited, environments beyond Earth.

\subsection{Timeline of Exploration}

With the Europa Clipper mission implementation well underway, we strongly endorse a new start for a Europa lander mission in the next decade. While Europa Clipper will provide critical reconnaissance data for landing site selection from both the scientific and engineering perspectives, a long delay between Europa Clipper's observations and a landed mission puts increased uncertainty on the state of Europa's possibly dynamic surface at landing, including locations of potential plumes or hotspots. We advocate that a landed mission is ready to follow Europa Clipper, offers civilization-scale science that engages many disciplines, and provides an exciting Flagship mission that performs in situ science on the surface of an Ocean World.

We envision a scenario in which the Europa lander mission is developed while Europa Clipper is en route to Jupiter, and launched approximately when Europa Clipper reaches Jupiter and begins its observations. This timing would allow at least five years to characterize potential landing sites on Europa's subjovian and antijovian hemispheres, before selection of a landing site. Programmatically, NASA's investments in Europa Clipper, the Europa Lander Mission Concept, and the landing systems developed for Mars Curiosity and Perseverance, position NASA to initiate the bold next step of landing on Europa in the next decade.

Looking beyond a lander, we also call for continued investment in drill and melt probe technologies in this decade, to allow for subsurface exploration and ocean access in future decades (Schmidt et al., 2020). In addition, we recommend that the Decadal Survey consider the critical role of team dynamics, equity, diversity, inclusion, and accessibility in planetary science. [Please see additional white papers on this topic.]

This Europa exploration strategy encompasses "civilization-scale science": The potential detection and characterization of life within an Ocean World in our very own Solar System would have profound implications for our understanding of the universe and our place in it. These are the inspirational goals called out in the NASA strategic science plan (NASA, 2018), and the exploration of Europa should be a key component of that strategy. 


\section{Summary and Recommendations}

We re-affirm that Europa is a target highly worthy of focused scientific exploration across a range of disciplines. With the Europa Clipper mission well into development, we anticipate a rich dataset to help characterize Europa's ocean, geology, chemistry, external environment, and potential habitability. We recommend that Europa Clipper be followed by a Europa Lander mission to be started in the decade covered by this survey. This should be only the first in a series of landed Ocean World missions that should later include a melt probe or subsurface access mission to directly study Europa's ocean. Finding life on Europa would be civilization-scale science, changing how we think about our place in the cosmos; a continued Europa exploration strategy could put that discovery within our reach for the first time in human history.

\section{References}

Anderson, J. D., et al. (1998). Science 281, 2019-2022. Balint, T. S., et al. (2020). Enabling the Next Frontiers in Astrobiology-Ocean and Ice Worlds Explorations with a Radioisotope Power System Inside a Pressure Vessel. White paper submitted to the 2020 Plan. Sci. \& Astrobio. Decadal Survey. Bierhaus, E. B., et al. (2009). In Europa, R. T. Pappalardo et al., eds., pp. 161-180. UA Press, Tucson. Carlson, R. W., et al. (2005), Icarus 177, 461-471. Carlson, R. W., et al. (2009). In Europa, R. T. Pappalardo et al., eds., pp. 283-327. UA Press, Tucson. Chyba, C. F., and C. B. Phillips (2001). PNAS 98(3), 801-804. Chyba, C. F., and C. B. Phillips (2007). Europa, in Planets and Life: The Emerging Science of Astrobiology, ed. W. Sullivan and J. Baross, Cambridge Univ. Press. Collins, G. C., et al. (2009). Planetary Tectonics 11(264), 229. COMPLEX, Committee on Planetary and Lunar Exploration, National Research Council (1999). A Science Strategy for the Exploration of Europa, National Academy Press, Washington, DC. Europa Lander Science Definition Team Report (2016). https://europa.nasa.gov/resources/58/europa-lander-study-2016-report/ Europa Lander Mission Concept Resources: https://www.jpl.nasa.gov/missions/europa-lander/ Gaidos, E. J., et al. (1999). Science 284 (5420), 1631-1633. Greeley, R., et al. (2000). Geologic mapping of Europa. JGR 105, 22,559-22,578. Hand, K. P., et al. (2007). Astrobiology 7(6), 1006-1022. Hand, K. P., et al. (2009). In Europa, R. T. Pappalardo et al., eds., pp. 589-629. UA Press, Tucson. Hand, K. P., et al. (2020a). On the Past, Present, and Future Role of Biology in NASA's Exploration of our Solar System. White paper submitted to the 2020 Plan. Sci. \& Astrobio. Decadal Survey. Hand, K. P., et al. (2020b). The Science of the Europa Lander mission concept. White paper submitted to the 2020 Plan. Sci. and Astrobio. Decadal Survey. Hansen, G. B., and T. B. McCord (2008). GRL 35, https://doi.org/10.1029/2007GL031748. Kattenhorn, S. A., \& Prockter, L. M. (2014). Nature Geoscience, 7(10), 762-767. Kuiper, G. P. (1957). Infrared Observations of Planets and Satellites. $A J$ 62, 245. McCord, T. B., et al. (2001). J. Geophys. Res. 106, E2, 3311-3319. Moore, W. B., \& Hussmann, H. (2009). In Europa, Pappalardo et al., eds., p. 369. UA Press, Tucson. NASA (2020). https://science.nasa.gov/science-red/s3fs-public/atoms/files/2020-2024_Science-TAGGED.pdf NASA (2018). https://www.nasa.gov/sites/default/files/atoms/files/nasa_2018_strategic_plan.pdf National Research Council (2003). New Frontiers in the Solar System: An Integrated Exploration Strategy. Washington, DC: The National Academies Press. https://doi.org/10.17226/10432. Oleson, S., et al. (2019). NASA/TP-2019-220054. Pappalardo, R.T., et al. (1999). J. Geophys. Res. 104, 24,01524,055. Pappalardo, R. T., et al. (2020). The Europa Clipper mission. White paper submitted to the 2020 Plan. Sci. \& Astrobio. Decadal Survey. Schubert, G., et al. (2009). In Europa, R. T. Pappalardo et al., eds., pp. 353-367. UA Press, Tucson. Schmidt, B., et al. (2020). Subsurface Needs for Ocean Worlds. White paper submitted to the 2020 Plan. Sci. \& Astrobio. Decadal Survey. Space Studies Board (2011). Vision and Voyages for Planetary Science in the Decade 2013-2022. Washington, D.C.: The National Academies Press. doi:10.17226/13117. Vance, S. D., et al. (2016). GRL 43(10), 4871-4879. Walker, C., et al., (2020). The Importance of Further Studies and Missions to Understand Cryovolcanism. White paper submitted to the 2020 Plan. Sci. \& Astrobio. Decadal Survey. 